\title{
FIXED POINT FREE INVOLUTIONS ON RIEMANN SURFACES
}

\author{
HUGO PARLIER
}

\begin{abstract}
Involutions without fixed points on hyperbolic closed Riemann surface are discussed. For an orientable surface $X$ of even genus with an arbitrary Riemannian metric $d$ admitting an involution $\tau$, it is known that $\min _{p \in X} d(p, \tau(p))$ is bounded by a constant which depends on the genus of $X$. The equivalent result is proved to be false in odd genus, and the optimal constant for hyperbolic Riemann surfaces is calculated in genus 2 .
\end{abstract}

\section{INTRODUCTION}

Involutions play an important role in the study of compact Riemann surfaces. For instance, the study of hyperelliptic surfaces of genus $g$ corresponds to the study of surfaces admitting an orientation preserving involution with $2 g+2$ fixed points and a Klein surface is the quotient of a Riemann surface by an orientation reversing involution. Furthermore, by the uniformization theorem, surfaces with an orientation reversing involution are conformally equivalent to real algebraic curves. A further motivation can be found in [1] where the so-called area filling conjecture is treated. The conjecture, first found in 4 for the $n$-dimensional case, states (in two dimensions) that any surface $S$ with a simple boundary, endowed with a Riemannian metric with the property that diametrally opposite points on the boundary are of distance $\geq \pi$, has area greater or equal to $2 \pi$, equality occurring only in the case of the classical hemisphere. The conjecture is equivalent to the following:

Conjecture Let $S$ be an orientable surface of even genus with a Riemannian metric $d$ that admits a fixed point free, orientation reversing involution $\tau$. Then there is a point $p \in S$ with

$$
\frac{d(p, \tau(p))^{2}}{\operatorname{area}(\mathcal{S})} \leq \frac{\pi}{4}
$$

Date: October 8, 2018.

2000 Mathematics Subject Classification. Primary 30F45; Secondary 30F20, 53C23.

Key words and phrases. Orientation reversing involutions, simple closed geodesics, hyperbolic Riemann surfaces.

The author was supported in part by the Swiss National Science Foundation grants 20 - 68181.02 and PBEL2-106180. 
In [1], this problem is solved when $S$ is hyperelliptic, thus in particular for genus 2. Furthermore, if $S$ is not hyperelliptic, in [5] it is shown that

$$
\frac{d(p, \tau(p))^{2}}{\operatorname{area}(\mathcal{S})} \leq C
$$

where $C \in\left[\frac{\pi}{4}, 1\right]$. If $S$ is a hyperbolic Riemann surface of even genus $g$, this shows that

$$
d(p, \tau(p)) \leq \sqrt{C 2 \pi(2 g-2)}
$$

The idea of the paper is to treat these questions in more detail for hyperbolic Riemann surfaces. The first main result of the article is to show that the conjecture stated above cannot be extended to odd genus, even if one restricts oneself to hyperelliptic surfaces.

Theorem 1.1. For any odd $g \geq 3$ and positive constant $k$, there exists a hyperbolic Riemann surface $S$ of genus $g$ admitting an orientation reversing involution $\tau$ which verifies $d(p, \tau(p))>k$ for all $p \in S$. Furthermore, $S$ can be chosen hyperelliptic.

The corresponding problem for orientation preserving involutions is also treated, with the same result.

The bound in equation 3 is not sharp and although the bound in 2 is sharp for arbitrary metrics, it is not sharp for hyperbolic metrics. The second main result of the article is the sharp bound in genus 2 for hyperbolic metrics.

Theorem 1.2. Let $S$ be a Riemann surface of genus 2 endowed with a hyperbolic metric and a fixed point free involution $\tau$. On $S$, there is a p such that $d(p, \tau(p)) \leq$ $\operatorname{arccosh}\left(\frac{5+\sqrt{17}}{2}\right)$. This upper-bound is sharp and is attained by a unique hyperbolic Riemann surface of genus 2 (up to isometry).

Compare the optimal bound $(=2.19 \ldots)$ with the bound from equation 3 (= $3.54 \ldots)$ applied to hyperbolic surfaces. The unique surface which attains this upper bound is surprisingly not the surface known as the Bolza curve, which is maximal for systole length, number of systoles (12), number of automorphisms (48), and verifies an optimal systolic inequality for hyperelliptic invariant CAT(0) metrics in genus 2 (see [10] and 6]).

\section{Definitions, notations And PRELIMINARies}

In this article, a surface will always mean a compact Riemann surface endowed with a hyperbolic metric. We shall suppose that the boundary of a surface is a collection of simple closed geodesics. The signature of the surface will be denoted $(g, n)$ (genus and number of boundary components) and verifies $(g, n) \geq(0,3)$ (with lexicographic ordering) and $(g, n) \neq(1,0)$. This condition is imposed by the existence of a hyperbolic metric. A surface of signature $(0,3)$ is commonly called a 
$Y$-piece, or a pair of pants. The area of the surface is given by area $(S)=-2 \pi \chi(S)$ where $\chi(S)=2-2 g-n$ is the Euler characteristic of the surface. Unless mentioned, a geodesic is a simple closed geodesic on $S$. Distance on $S$ (between subsets, points or curves) is denoted $d(\cdot, \cdot)$. Curves and geodesics will be considered primitive and non-oriented, and can thus be seen as point sets on $S$. A geodesic $\gamma$ (resp. a set of geodesics $E$ ) is called separating if $S \backslash \gamma$ (resp. $S \backslash E$ ) is not connected. Let us recall that a non-trivial closed curve $c$ (not necessarily simple) on $S$ is freely homotopic to exactly one closed geodesic $\gamma$. (We denote $\mathcal{G}(c)=\gamma$ ). If $c$ is simple, then so is $\gamma$. We shall denote the intersection number of two geodesics $\gamma$ and $\delta$ by $\operatorname{int}(\gamma, \delta)$. The length of a path or a curve will be denoted $\ell(\cdot)$, although in general a curves name and its length will not be distinguished. The systole $\sigma$ of a surface is the (or a) shortest non-trivial closed curve on $S$ (sometimes called a systolic loop, although for Riemann surfaces, systole seems to be the standard denomination). A systole is always a simple closed geodesic and cannot intersect another systole more than once. An involution $\tau$ is an isometric automorphism of the surface that is of order 2 . Involutions can either be orientation preserving or not. For Riemann surfaces this is equivalent to whether the involution is holomorphic or antiholomorphic. Let us also recall that two geodesics of length less or equal to 2 arcsinh1 are disjoint (and simple). This can be seen using the following well known result, commonly called the collar theorem (i.e. [7, [2], [3, 9]).

Theorem 2.1. Let $\gamma_{1}$ and $\gamma_{2}$ be non-intersecting simple closed geodesics on $S$. Then the collars

of widths

$$
\mathcal{C}\left(\gamma_{i}\right)=\left\{p \in S \mid d_{S}\left(p, \gamma_{i}\right) \leq w\left(\gamma_{i}\right)\right\}
$$

$$
w\left(\gamma_{i}\right)=\operatorname{arcsinh}\left(1 / \sinh \frac{\gamma_{i}}{2}\right)
$$

are pairwise disjoint for $i=1,2$. Furthermore, each $\mathcal{C}\left(\gamma_{i}\right)$ is isometric to the cylinder $\left[-w\left(\gamma_{i}\right), w\left(\gamma_{i}\right)\right] \times \mathbb{S}^{1}$ with the metric $d s^{2}=d \rho^{2}+\gamma_{i}^{2} \cosh ^{2} \rho d t^{2}$.

Notice that the $\gamma_{i}$ 's divide their collar into two connected spaces which we will call half-collars. In the sequel we will make use of the fact that the collars of two disjoint geodesics are also disjoint.

Simple closed geodesics and orientation reversing involutions are closely related. The following proposition is an extension of what is generally called Harnack's theorem [11] and can be found in [8].

Proposition 2.2. If a surface $S$ admits $\tau$, an orientation reversing involution, then the fixed point set of $\tau$ is a set of $n$ disjoint simple closed geodesics $\mathcal{B}=\left\{\beta_{1}, \ldots, \beta_{n}\right\}$ with $n \leq g+1$. In the case where the set $\mathcal{B}$ is separating, then $S \backslash \mathcal{B}$ consists of two connected components $S_{1}$ and $S_{2}$ such that $\partial S_{1}=\partial S_{2}=\mathcal{B}$ and $S_{2}=\sigma\left(S_{1}\right)$. If not, then $\mathcal{B}$ can be completed by either a set $\alpha$ which consists of one or two simple closed geodesics such that $\mathcal{B} \cup \alpha$ has the properties described above (with the important difference that $\alpha$ does not contain any fixed points of $\sigma$ ). Each of the simple closed geodesics in $\alpha$ is globally fixed by $\sigma$. 


\section{The General CASE}

Let $S$ be a surface of genus $g \geq 2$, endowed with a hyperbolic metric and a fixed point free involution $\tau$.

Proposition 3.1. If $g$ is even, then $\tau$ is orientation reversing.

Proof. The proof is a consequence of the Riemann-Hurwitz formula, or can be seen as follows. In general, if $\tau$ is an orientation preserving isometry, then $S / \tau=O$ is an orientable orbifold with singular points who lift on $S$ to fixed points of $\tau$. As $\tau$ is without fixed points, $O$ is an orientable closed surface of genus $g_{o}$ endowed with a hyperbolic metric and thus of area $\frac{\operatorname{area}(S)}{2}$. This implies that $O$ 's Euler characteristic is equal to $(g-1)$. From this we have $g_{o}=\frac{g-1}{2}$ which is not possible if $g$ is even.

Remark 3.2. As the essence of the proof is purely topological, the proposition holds for arbitrary metric.

The following propositions concern further relationships between simple closed geodesics and involutions. Suppose that $S$ is a hyperbolic surface of genus $g \geq 2$ and $\tau$ is a fixed point free involution.

Proposition 3.3. Let $\sigma$ be a systole of $S$. Then $\sigma \cap \tau(\sigma)=\emptyset$ or $\sigma=\tau(\sigma)$.

Proof. Suppose that $\sigma \neq \tau(\sigma)$. As $\tau(\sigma)$ is necessarily another systole of $S$, then the curves $\sigma$ and $\tau(\sigma)$ cannot intersect more than once (this would imply the existence of a shorter non-trivial closed curve on $S$ ). Suppose that $\sigma \cap \tau(\sigma)=p$, where $p$ is a point. Then $\tau(p)=p$ which contradicts the hypotheses.

As $S$ is compact, the value $\min _{p \in S} d(p, \tau(p))$ exists and is attained for at least one point.

Proposition 3.4. Let $p \in S$ such that $d(p, \tau(p))$ is minimum. Then $p$ lies on a simple closed geodesic $\gamma$ of length $2 d(p, \tau(p))$ that verifies $\gamma=\tau(\gamma)$.

Proof. Let $p$ be such a point. Let $c_{p}$ be a minimal path between $p$ and $\tau(p)$ (thus $\left.d(p, \tau(p))=\ell\left(c_{p}\right)\right)$. Notice that $c_{p} \cap \tau\left(c_{p}\right)=\{p, \tau(p)\}$, and the two paths $c_{p}$ and $\tau\left(c_{p}\right)$ are not freely homotopic among simple paths with endpoints on $p$ and $\tau(p)$. Thus $c_{p} \cup \tau\left(c_{p}\right)$ is a non-trivial simple closed curve. Furthermore, it follows that $\gamma=\tau\left(\mathcal{G}\left(c_{p} \cup \tau\left(c_{p}\right)\right)=\mathcal{G}\left(c_{p} \cup \tau\left(c_{p}\right)\right)\right.$ and thus for $q \in \gamma, d(q, \tau(q)) \leq d(p, \tau(p))$ with equality occurring only in the case where $\mathcal{G}\left(c_{p} \cup \tau\left(c_{p}\right)\right)=c_{p} \cup \tau\left(c_{p}\right)$. This implies that $p$ lies on $\gamma$ and that $\gamma$ is of length $2 d(p, \tau(p))$.

Notice that for such a geodesic $\gamma$, all point are diametrically opposite to their images by $\tau$. In fact this is true for any simple geodesic left invariant by $\tau$.

Proposition 3.5. Let $\gamma$ be a simple closed geodesic such that $\tau(\gamma)=\gamma$. Then the image $\tau(p)$ of $p \in \gamma$ is the point on $\gamma$ diametrically opposite from $p$. 
Proof. For any $p \in \gamma$, the points $p$ and $\tau(p)$ separate $\gamma$ into two geodesic arcs. The image of one of the arcs has to be the other arc (otherwise $\tau$ has fixed points) and since $\tau$ is an isometry, the result follows.

As mentioned in the introduction, a result in [5] implies that for any $S$ of even genus with an involution $\tau$ (necessarily orientation reversing by proposition 3.1)

$$
d(p, \tau(p)) \leq \sqrt{C 2 \pi(2 g-2)}
$$

with $C \in\left[\frac{\pi}{2}, 2\right]$. This is false in odd genus for both orientation preserving and reversing involutions.

Theorem 3.6. Let $g \geq 3$ be an odd integer and $k$ a positive constant. There exists a hyperbolic Riemann surface $S$ of genus $g$ admitting an orientation preserving involution $\tau$ which verifies $d(p, \tau(p))>k$ for all $p \in S$. The same result holds for orientation reversing involutions. Furthermore, in both cases, $S$ can be chosen hyperelliptic.

Proof. We shall begin by showing the general idea for constructing surfaces with orientation preserving involutions, then surfaces with orientation reversing involutions which verify the conditions of the theorem. Finally, we shall give an explicit example of a hyperelliptic surface which has both an orientation preserving involution, and an orientation reversing involution which verifies the conditions of the theorem.

Consider a surface $\tilde{S}$ of signature $(\tilde{g}, 2)$ with boundary geodesics $\alpha$ and $\beta$ of equal length $x$. Let $p_{\alpha}$ and $q_{\alpha}$ be two diametrically opposite points on $\alpha$, and $p_{\beta}$ and $q_{\beta}$ be two diametrically opposite points on $\beta$. Consider two copies of $\tilde{S}$, say $\tilde{S}_{1}$ and $\tilde{S}_{2}$, and paste them along their boundary geodesics such that $\tilde{S}_{1}$ 's $\alpha$ is pasted to $\tilde{S}_{2}$ 's $\beta$, and $\tilde{S}_{2}$ 's $\alpha$ is pasted to $\tilde{S}_{1}$ 's $\beta$. The pasting must respect the choice of $p$ s and $q$ s, meaning $p_{\alpha}$ is pasted to $p_{\beta}$ etc. The resulting surface $S$ is an orientable surface of genus $2 g+1$ admitting an orientation preserving involution $\tau_{o}$ without fixed points which acts as follows: a point originally on $\tilde{S}_{1}$ is sent to it's corresponding point on $\tilde{S}_{2}$ and vice-versa. The simple closed geodesics of $S$, previously the boundary geodesics of $\tilde{S}_{1}$ and $\tilde{S}_{2}$, are of length $x$ and are reversed by $\tau_{o}$.

The image $\tau_{o}(p)$ of a point $p \in S$ is at least "half a collar away" from $p$, and by the collar theorem the following inequality is thus verified:

$$
d\left(p, \tau_{o}(p)\right)>\operatorname{arcsinh}\left(1 / \cosh \left(\frac{x}{2}\right)\right) .
$$

The half-collar length tends to infinity as $x$ tends to 0 , thus for any $k>0$, it suffices to chose $x$ such that $k<\operatorname{arcsinh}\left(1 / \cosh \left(\frac{x}{2}\right)\right.$, and the result follows.

Now let us treat the case of orientation reversing involutions. Consider a $\tilde{S}$ as above. Instead of pasting two identical copies of $\tilde{S}$, consider $\tilde{S}$ and a symmetric copy of $\tilde{S}$ (a mirror image), say $\tilde{S}_{-}$. Denote by $\alpha_{-}$and $\beta_{-}$the images of $\alpha$ and $\beta$ on $\tilde{S}_{-}$as in the following figure.

Paste the boundary geodesics together ( $\alpha$ to $\alpha_{-}, \beta$ to $\beta_{-}$) while respecting the choice of $p$ s and $q \mathrm{~s}$. The resulting surface $S$ is of genus $2 g+1$ and the orientation 

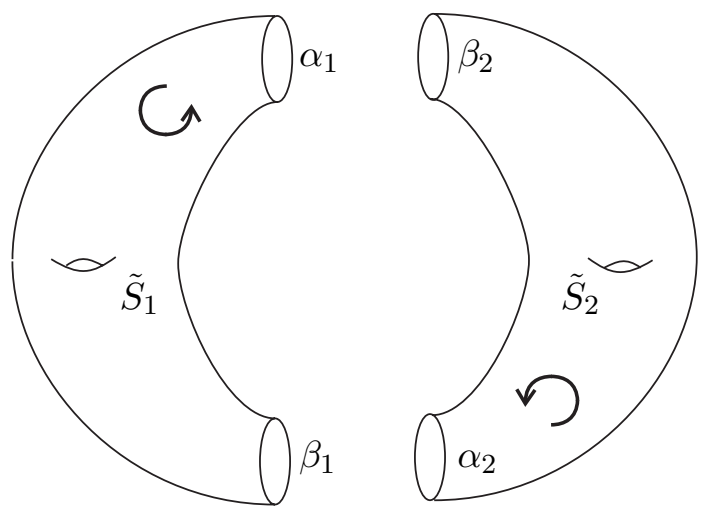

FiguRE 1. Example in genus 3 with orientation preserving involution
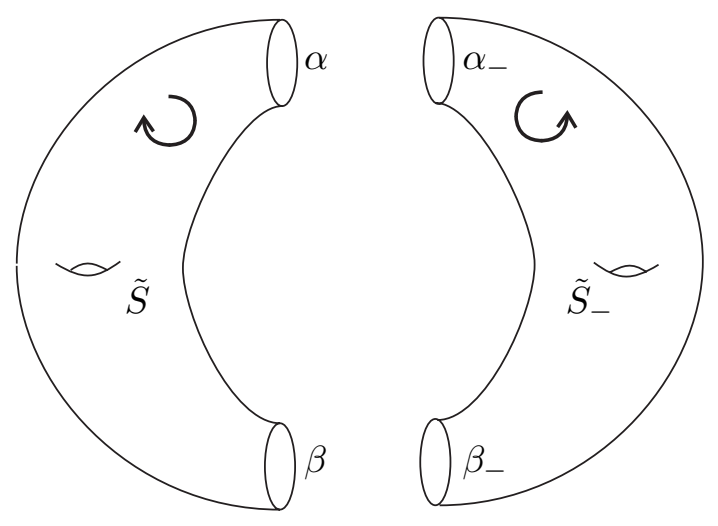

FiguRE 2. Example in genus 3 with orientation reversing involution

reversing involution $\tau_{r}$ is the isometry taking a point of $\tilde{S}$ to its corresponding point on $\tilde{S}_{-}$and vice-versa. As in the first examples, for any $k, x$, the length of both $\alpha$ and $\beta$, can be chosen such that $d\left(p, \tau_{r}(p)\right)>k$ for all $p \in S$.

We shall now give an explicit example of a hyperelliptic surface with both an orientation preserving involution and an orientation reversing involution which verifies the conditions of the theorem.

Consider a right-angled $2 g+4$-gon in the hyperbolic plane, say $P$, with edges labeled in cyclic ordering $\left\{a_{1}, b_{1}, \ldots, a_{g+2}, b_{g+2}\right\}$. The figures are all done when $g=3$. 


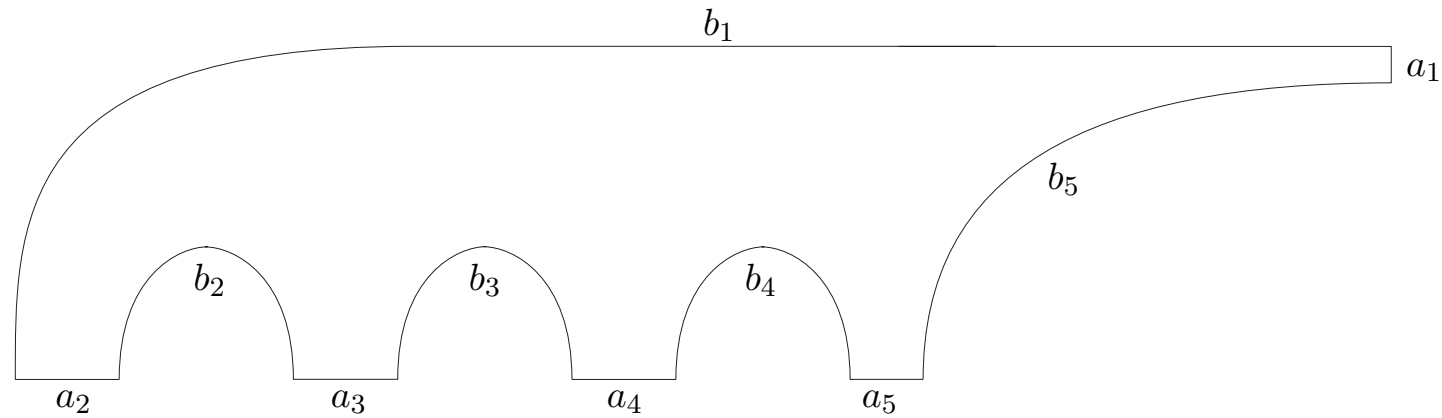

Figure 3 . The polygon $P$

Take two isometric copies of $P$, say $P_{1}$ and $Q_{1}$, and two symmetric images of $P$, say $P_{2}$ and $Q_{2}$ and paste them along the sides labeled $a_{i}$ as in the following figure to obtain a surface $S^{+}$of signature $(0,2 g+2)$. The bold curves represent the boundary geodesics of $S^{+}$.

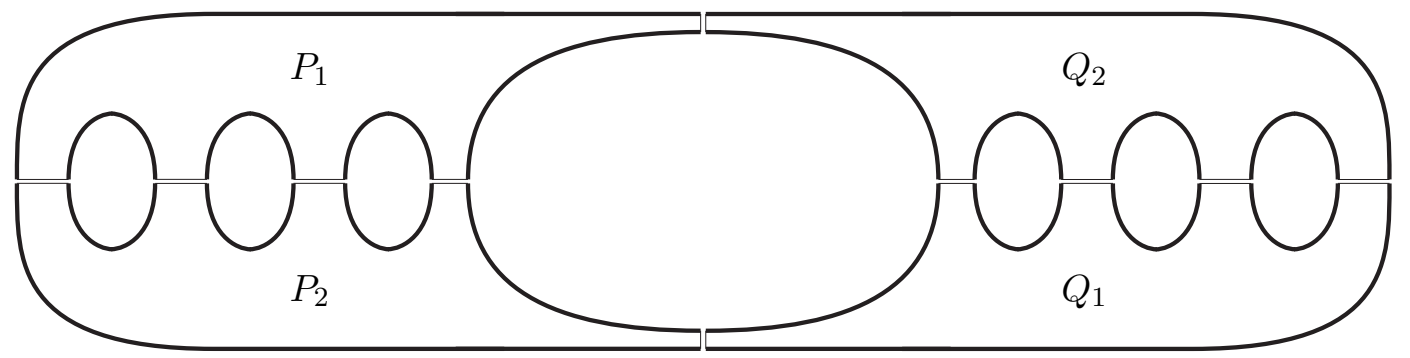

FiguRE 4 . The surface $S^{+}$

By taking a symmetric copy $S^{-}$of the surface thus obtained, and denote by $P_{1}^{-}$ etc. the various images of the polygons of $S^{+}$. By pasting the two surfaces $S^{+}$and $S^{-}$along the $2 g+2$ boundary geodesics, as in the following figure, one obtains a surface $S$ of genus $2 g+1$. We require the pasting to be exact - each edge of a polygon is pasted exactly to its corresponding edge with end points of each edge coinciding.

The surface thus obtained necessarily admits a number of involutions. One of these is the hyperelliptic involution $\tau_{h}$, with fixed points the end points of all the images of $a_{3}, \ldots, a_{g+1}$. (To be precise, $\tau_{h}$ exchanges $P_{1}$ and $P_{2}^{-}, P_{2}$ and $P_{1}^{-}, Q_{1}$ and $Q_{2}^{-}, Q_{2}$ and $Q_{1}^{-}$.)

The remaining involutions we are interested in are the ones without fixed points. The orientation preserving involution $\tau_{o}$ defined by exchanging $P_{1}$ with $Q_{1}, P_{2}$ with $Q_{2}, P_{1}^{-}$with $Q_{1}^{-}$, and $P_{2}^{-}$with $Q_{1}^{-}$, does not have any fixed points. The orientation reversing involution $\tau_{r}$ defined by exchanging $P_{1}$ with $Q_{1}^{-}, P_{2}$ with $Q_{2}^{-}, P_{1}^{-}$with 


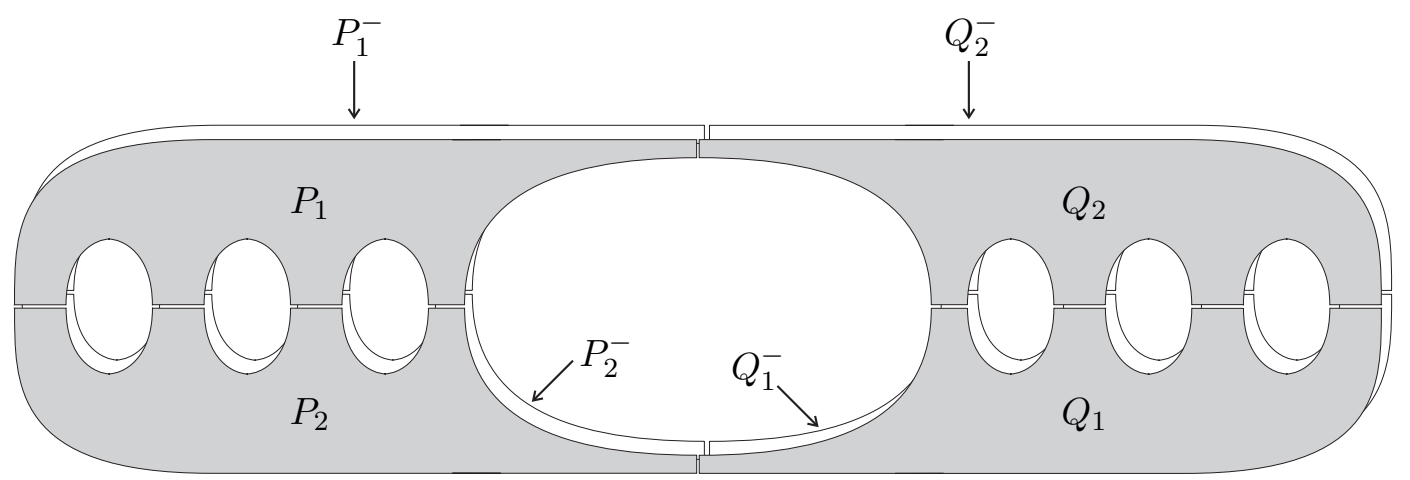

Figure 5. The surface $S$

$Q_{1}$, and $P_{2}^{-}$with $Q_{1}$, does not have any fixed points either. The eight images of the edge $a_{1}$ on $S$ form two simple closed geodesics (after pasting) of length $2 a_{1}$. As in the more general case, described above, for any $k$, we can chose a sufficiently short $a_{1}$ such that we have $d\left(p, \tau_{o}(p)\right)>k$ as well as $d\left(p, \tau_{r}(p)\right)>k$ for all $p \in S$.

\section{The CASE of Genus 2}

By the previous theorem, for all surfaces of genus 2, the minimum distance between a point and the image by an involution is bounded. The object of this section is a detailed study of fixed point free involutions on surfaces of genus 2 leading to the sharp bound on this distance.

Lemma 4.1. Let $\tau$ be an involution without fixed points on a surface of genus 2 . Then the following statements are true:

(1) $\tau$ reverses orientation.

(2) $S$ contains a separating simple closed geodesic $\beta$ such that $\beta=\tau(\beta)$. The two parts of $S$ separated by $\beta$ are interchanged by $\tau$.

(3) For any $p \in \beta, p$ and $\tau(p)$ are diametrically opposite.

Proof. Parts (1) and (3) have been treated earlier in the general case and part (2) is a direct consequence of proposition 2.2 (Harnack's theorem).

The geodesic $\beta$ from the previous lemma divides $S$ into two surfaces of signature $(1,1)$. The following lemma recalls some essential facts about these surfaces. These facts are either well known, or their proofs can be found in [10] (theorem 4.2, p. 578). For such surfaces, and a choice of interior simple closed geodesic $\alpha$, we denote $h_{\alpha}$ the unique simple geodesic path which goes from boundary to boundary and intersects boundary at two right angles and does not cross $\alpha$. We will refer to the geodesic path $h_{\alpha}$ as the height associated to $\alpha$. 


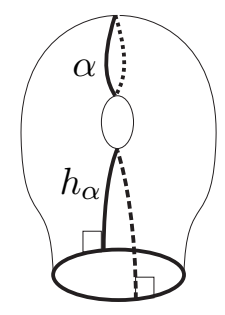

Figure 6 . The curve $h_{\alpha}$ associated to $\alpha$

Lemma 4.2. Let $Q$ be a surface of signature $(1,1)$ with boundary geodesic $\beta$. Then the following statements are true:

(1) $Q$ is hyperelliptic and its hyperelliptic involution has three fixed points in the interior of $Q$ (the Weierstrass points of $Q$ ).

(2) Let $\gamma$ be an interior simple closed geodesic of $Q$ and denote its associated height $h_{\gamma}$. Then $\gamma$ passes through exactly two of the three Weierstrass points and the remaining Weierstrass point is the midpoint of $h_{\gamma}$. Furthermore, the length of $\gamma$ is directly proportional to the length of $h_{\gamma}$.

(3) Among all surfaces of boundary length $\ell(\beta)$, the unique surface (up to isometry) with maximum length systole is the surface with three distinct systoles.

(4) If $Q$ has three systoles, then their associated heights do not intersect and are evenly spaced along $\beta$.

We shall now proceed to the main result of this section.

Theorem 4.3. Let $S$ be a Riemann surface of genus 2 endowed with a hyperbolic metric and a fixed point free involution $\tau$. On $S$, there is a $p$ such that $d(p, \tau(p)) \leq$ $\operatorname{arccosh}\left(\frac{5+\sqrt{17}}{2}\right)$. This upper-bound is sharp and is attained by a unique hyperbolic Riemann surface of genus 2 (up to isometry).

Proof. For $S$ of genus 2, let $\beta$ be the separating geodesic described in the previous lemma 4.1] Let $Q$ be one of the two surfaces of signature $(1,1)$ separated by $\beta$. Proposition 3.4 implies that a $p$ which minimizes $d(p, \tau(p)$ is found on a simple closed geodesic left invariant by $\tau$. As all simple closed geodesics in the interior of both $Q$ and $\tau(Q)$ are distinct from their images, $p$ must be found on a simple geodesic that either crosses $\beta$ or is $\beta$.

Let $h$ be a height on $Q$. Then $h \cup \tau(h)=\gamma_{h}$ is a simple closed geodesic with the property that for $p \in \gamma_{h}, \tau(p)$ is diametrically opposite to $p$ (lemma 4.1). Furthermore, if $\sigma$ is a systole on $Q$, then $h_{\sigma}$ is the shortest height on $Q$. Notice that all simple closed geodesics that cross $\beta$ are longer that $2 h_{\sigma}$, thus $d(p, \tau(p)) \geq h_{\sigma}$, equality occurring when $2 h_{\sigma} \leq \beta$. For any $\beta$, the maximum value that $h_{\sigma}$ can attain is attained in the situation described in lemma 4.2 . Furthermore, the maximum value 
of $h_{\sigma}$ is strictly proportional to the length of $\beta$. The maximum value for $d(p, \tau(p))$ is thus obtained when both $\beta / 2$ and maximum $h_{\sigma}$ are equal. These conditions define a unique surface of signature $(1,1)$, whose lengths verify (i.e. [10])

$$
4 \cosh ^{3}\left(\frac{\sigma}{2}\right)+6 \cosh ^{2}\left(\frac{\sigma}{2}\right)+1=\cosh \left(\frac{\beta}{2}\right)
$$

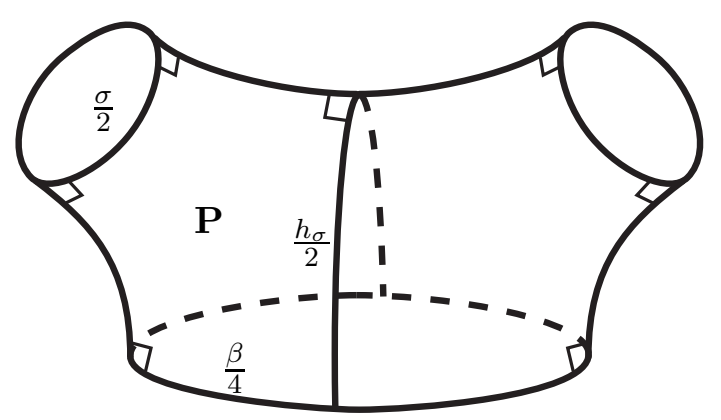

Figure 7. Maximal $Q$ cut along a systole

The figure shows this surface after cutting along a systole $\sigma$. The hyperbolic right angled pentagon $\mathbf{P}$ has equal length adjacent edges of lengths $\frac{h_{\sigma}}{2}$, and $\frac{\beta}{4}$, and opposite edge of length $\frac{\sigma}{2}$. Using the hyperbolic trigonometry formula for such pentagons, one obtains

and thus

$$
\sinh ^{2}\left(\frac{\beta}{4}\right)=\cosh \left(\frac{\sigma}{2}\right)
$$

$$
\cosh \left(\frac{\beta}{2}\right)-1=2 \cosh \left(\frac{\sigma}{2}\right)
$$

Using equations 4 and 5 , the value for a systole $\sigma$ verifies

$$
2 \cosh ^{2}\left(\frac{\sigma}{2}\right)-3 \cosh \left(\frac{\sigma}{2}\right)-1=0
$$

From this we can deduce the value

$$
h_{\sigma}=\frac{\beta}{2}=\operatorname{arccosh}\left(\frac{5+\sqrt{17}}{2}\right) \text {. }
$$

In order to obtain the maximal surface of genus 2 , it suffices to take two copies of the maximum surface of signature $(1,1)$ and to paste them such that minimum length heights touch. As the heights are evenly spaced along $\beta$, whichever way this is done one will obtain the same surface. This surface, say $S_{\max }$, is clearly unique up to isometry. The following figure explicitly illustrates how to obtain $S_{\max }$ by 
pasting 8 copies of $\mathbf{P}$. On $\mathbf{P}$, consider the midpoint of the edge labeled $\frac{\sigma}{2}$ on figure 7. The points labeled $p_{1}, p_{2}, q_{1}$ and $q_{2}$ are the 8 copies of this point and the points $p_{3}$ and $q_{3}$ are as labeled on the figure. The pasting of the boundary geodesics is as indicated in the figure ( $p_{1}$ pasted to $p_{1}$ etc.). The Weierstrass points of $S_{\max }$ are exactly the points $p_{1}, p_{2}, p_{3}$ and $q_{1}, q_{2}, q_{3}$.

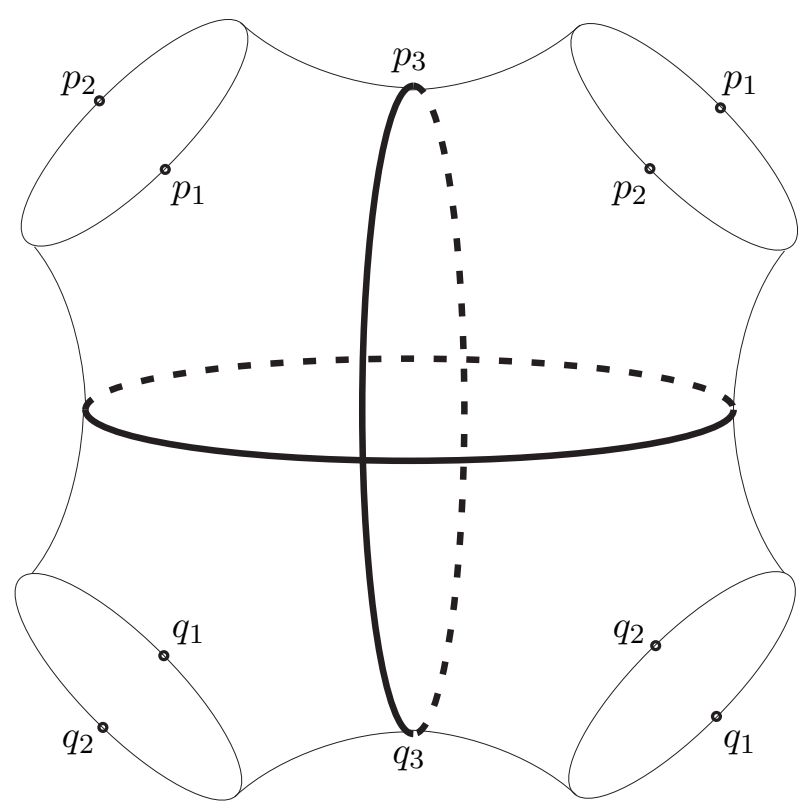

FiguRe $8 . S_{\max }$

Notice that their are exactly 6 systoles on this surface, and the length of a systole of the surface is the same as a systole on the maximal $(1,1)$ surface. The length of a systole, by equation [6] is

$$
\sigma=2 \operatorname{arccosh}\left(\frac{3+\sqrt{17}}{4}\right)
$$

The maximal surface $S_{\max }$ we have constructed above has a remarkable property: it is not the Bolza curve. The Bolza curve, with its unique hyperbolic metric, is constructed in the same fashion, namely it is obtained by pasting two maximal surfaces of signature $(1,1)$. It is the unique maximal genus 2 surface for systole length. Compared to $S_{\max }$, its separating geodesic $\beta$ is longer and its systole length is $2 \operatorname{arccosh}(1+\sqrt{2})$.

\section{REFERENCES}

[1] V. Bangert, C. Croke, S. V. Ivanov, and M. G. Katz. Filling area conjecture and ovalless real hyperelliptic surfaces. Geom. Funct. Anal., to appear. 
[2] Peter Buser. The collar theorem and examples. Manuscripta Math., 25(4):349-357, 1978.

[3] Peter Buser. Geometry and spectra of compact Riemann surfaces, volume 106 of Progress in Mathematics. Birkhäuser Boston Inc., Boston, MA, 1992.

[4] Mikhael Gromov. Filling Riemannian manifolds. J. Differential Geom., 18(1):1-147, 1983.

[5] Sergei V. Ivanov and Mikhail G. Katz. Generalized degree and optimal Loewner-type inequalities. Israel J. Math., 141:221-233, 2004.

[6] M. G. Katz and S. Sabourau. An optimal systolic inequality for cat(0) metrics in genus 2. available on the arxiv:math.DG/0501017 2005.

[7] Linda Keen. Collars on Riemann surfaces. In Discontinuous groups and Riemann surfaces (Proc. Conf., Univ. Maryland, College Park, Md., 1973), pages 263-268. Ann. of Math. Studies, No. 79. Princeton Univ. Press, Princeton, N.J., 1974.

[8] Irwin Kra and Bernard Maskit. Bases for quadratic differentials. Comment. Math. Helv., 57(4):603-626, 1982.

[9] Burton Randol. Cylinders in Riemann surfaces. Comment. Math. Helv., 54(1):1-5, 1979.

[10] P. Schmutz. Riemann surfaces with shortest geodesic of maximal length. Geom. Funct. Anal., 3(6):564-631, 1993.

[11] G. Weichold. Ueber symmetrische Riemann'sche Flchen und die Periodicittsmoduln der zugehrigen Abel'schen Normalintegrale erster Gattung. PhD thesis, Diss. Leipzig. Schlmilch Z. XXVIII. 321-352. , 1883.

Departamento de Matemáticas Fundamentales, Facultad de Ciencias, Universidad NACional De Educación a Distancia, Madrid 28040, SPAIN

E-mail address: hugo.parlier@epfl.ch 